\title{
Cosmic ray flux and lockdown due to COVID-19 in Kolkata - Any correlation?
}

\author{
A SEN, S CHATTERJEE, S ROY, R BISWAS, S DAS, S K GHOSH and S BISWAS $\oplus^{*}$ \\ Department of Physics and Centre for Astroparticle Physics and Space Science, Bose Institute, EN-80, Sector V, \\ Kolkata 700 091, India \\ *Corresponding author. E-mail: saikat@jcbose.ac.in; saikat.ino@gmail.com
}

MS received 20 November 2020; revised 30 December 2020; accepted 5 January 2021

\begin{abstract}
Cosmic ray muon flux is measured by the coincidence technique using plastic scintillation detectors in the High Energy Physics Detector Laboratory at Bose Institute, Kolkata. Due to the COVID-19 outbreak and nationwide complete lockdown, the laboratory was closed from the end of March 2020 till the end of May 2020. After lockdown, although the city is not in its normal state, we still were able to take data on some days. The lockdown imposed a strict restriction on the transport service other than the emergency ones and also most of the industries were shut down in and around the city. This lockdown has significant effect on the atmospheric conditions in terms of change in the concentration of air pollutants. We have measured the cosmic ray flux before and after the lockdown to observe the apparent change if any, due to change in the atmospheric conditions. In this article, we report the measured cosmic ray flux at Kolkata $\left(22.58^{\circ} \mathrm{N} 88.42^{\circ} \mathrm{E}\right.$ and $11 \mathrm{~m}$ above the Sea Level) along with the major air pollutants present in the atmosphere before and after the lockdown.
\end{abstract}

Keywords. Cosmic ray; muon flux; plastic scintillation detector; air quality index; air pollutants.

PACS Nos 29.40.Mc; 95.55.Vj; 95.85.Ry; 96.40.-z; 07.89.+b

\section{Introduction}

Cosmic ray consists of high-energy particles that mostly originate from the outer space, with some very high energy particles which can have extragalactic origin. Primary cosmic rays consist of $90 \%$ protons, $9 \% \alpha-$ particles and other heavier nuclei [1]. These primary cosmic rays interact with the gas molecules in the atmosphere and produce secondary cosmic rays. These secondary particles consist mostly of pions and some kaons. Neutral pions $\left(\pi^{0}\right)$ decay into $\gamma$-rays that generate electromagnetic showers $\left(e^{+}, e^{-}, \gamma\right)$, which possess low penetration power. Charged pions $\left(\pi^{+}, \pi^{-}\right)$decay into muons and neutrinos. Neutrinos have a very small cross-section for interaction and typically pass through the Earth without any further interactions. On the other hand, muons are heavy particles and thus loss of energy through bremsstrahlung is negligible for them. This makes the muon a very penetrating particle, unlike electron. The muon has a lifetime of $2.2 \mu \mathrm{s}$, yet it still makes it down to detectors at the surface of the Earth traversing through the atmosphere. This is because muons travels at a speed that is close to that of light and thus experience relativistic time dilation and therefore can be detected by our detectors. Since the secondary cosmic rays are mostly muons and they can travel large distance through the atmosphere before they are detected, it will be really interesting if any correlation of this cosmic ray muon flux with the change in atmosphere in terms of the concentration of air pollutants is found [2-4].

For this study, cosmic ray flux has been measured in our laboratory using plastic scintillator detectors before and after the imposition of lockdown due to the COVID19 pandemic. The effects of atmospheric pressure and temperature on the muon flux have also been studied here. A brief description of the change of the atmospheric parameters due to lockdown is discussed in the next section. The succeeding sections describe the details of the experimental set-up and results, followed by summary and discussions.

\section{Effect of lockdown}

India is at a critical stage in its fight against COVID-19 with positive cases crossing $89,58,140$ and death toll at 


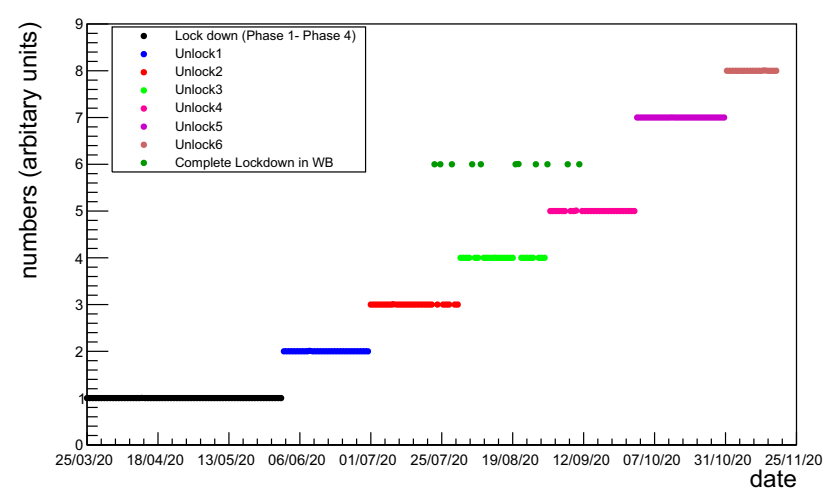

Figure 1. Different phases of lockdown and unlock as a function of date. The complete lockdown is marked with 1 , different unlock phases are marked as 2, 3 so on and the complete lockdown days in West Bengal during the unlock phases are marked with 6 .

1,31,618 until November 18, 2020 [5]. The entire country was under complete lockdown from March 25 to April 14, 2020, for 21 days, which was further extended by the Government of India till May 3, 2020, followed by the third phase of lockdown till May 17, 2020, and the fourth phase till May 31, 2020, to tackle the spread of COVID-19. Restrictions on social gathering and travelling resulted in the shutdown of all the businesses which include industries, transport (air, water and surface), markets, shops, tourism, construction and demolition, hotels and restaurants, mining and quarrying, etc. except essential services like groceries, milk, medicines and emergency services like hospital, fire service and administration. In June 2020, both central and state governments declared restricted unlocking phase. While unlock phases started from 1 June 2020, there were complete lockdown in West Bengal on some selected dates to fight against COVID-19. The dates of lockdown are mentioned in figure 1 .

The complete lockdown is marked with 1 , different unlock phases are marked as 2, 3 so on and the complete lockdown days in West Bengal during the unlock phases are marked with 6. During the lockdown (25 March-6 April 2020; Lockdown Phase-1) and before lockdown (10-20 March 2020), significant variation in the concentrations of the five most abundant pollutants in the air $\left(\mathrm{PM}_{2.5}, \mathrm{PM}_{10}, \mathrm{NO}_{2}, \mathrm{CO}, \mathrm{O}_{3}\right)$ are observed in Kolkata. The concentrations of air pollutants in Kolkata are decreased by $\sim 23 \%\left(\mathrm{PM}_{2.5}\right)$, $\sim 34 \%\left(\mathrm{PM}_{10}\right), \sim 60 \%\left(\mathrm{NO}_{2}\right), \sim 29 \%$ (CO) while the $\mathrm{O}_{3}$ concentration is increased by $\sim 17 \%$ due to the clearer atmosphere compared to that before the lockdown period [2]. We also looked into the last year data for the same period (March-April, 2019) and found that, during the lockdown, the concentrations of air pollutants are decreased by $\sim 27 \%\left(\mathrm{PM}_{2.5}\right), \sim 32 \%\left(\mathrm{PM}_{10}\right), \sim 66 \%$
Pramana-J. Phys.

(2021) $95: 64$

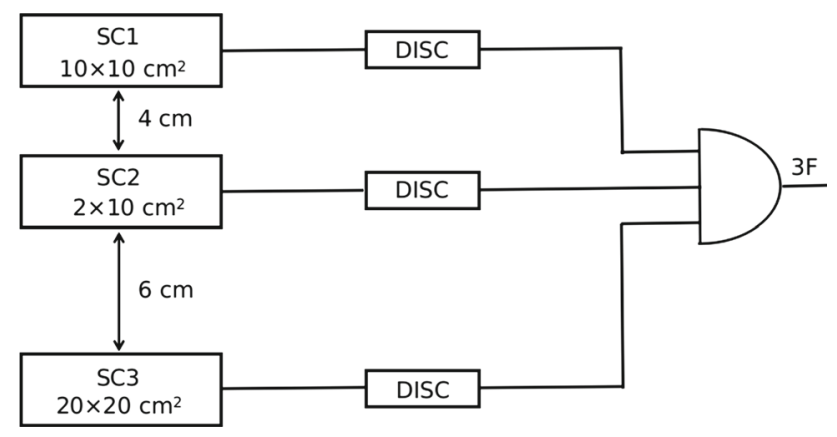

Figure 2. Schematic of the experimental set-up for muon flux measurement at the laboratory.

$\left(\mathrm{NO}_{2}\right), \sim 16 \%(\mathrm{CO})$ and $\mathrm{O}_{3}$ concentration is increased by $\sim 87 \%$ [2].

We have used the live day-to-day data from ref. [6] of the concentrations of the seven major air pollutants and studied their effects on cosmic ray flux. In our work, we have reported the measured muon fluxes before and after the lockdown at Kolkata and tried to correlate the same with the change in concentrations not only of the individual components of air pollutants but also of the total amount of pollutants.

\section{Experimental set-up}

The schematic of the muon flux measurement set-up is shown in figure 2. Three plastic scintillators tagged as SC1, SC2 and SC3, made using BC400 material are used in this set-up [7,8]. The dimensions of these scintillators are $10 \times 10 \mathrm{~cm}^{2}, 2 \times 10 \mathrm{~cm}^{2}$ and $20 \times 20 \mathrm{~cm}^{2}$ respectively. The coincidence area of the three detectors is $20 \mathrm{~cm}^{2}$. The distance between the top and bottom scintillators is $\sim 10 \mathrm{~cm}$ whereas that between the top and the middle one is $4 \mathrm{~cm}$. Each scintillator is connected with a photomultiplier tube (PMT) and a base where one SHV (safe high voltage) and one BNC (Bayonet NeillConcelman) connectors are provided for the application of high voltage (HV) and collection of signals respectively. A voltage of $+1550 \mathrm{~V}$ is applied to all the PMTs. Thresholds to the discriminators are set to $-15 \mathrm{mV}$ for all the scintillators. The width of each discriminator output is kept at $50 \mathrm{~ns}$. The coincidence of these three signals is achieved using a logic unit. The three-fold coincidence signal is then counted using a scaler and then divided by the product of the area of coincidence window $\left(20 \mathrm{~cm}^{2}\right)$, muon detection efficiency of the system $(\sim 72 \%)$ [8] and the measurement time to get the muon flux. The calculated muon flux is then multiplied by a factor of 0.95 to correct the effect of the difference in the threshold settings to the discriminator for the scintillators, during the efficiency measurement [8] (threshold is $-30 \mathrm{mV}$ ) and the present measurement 


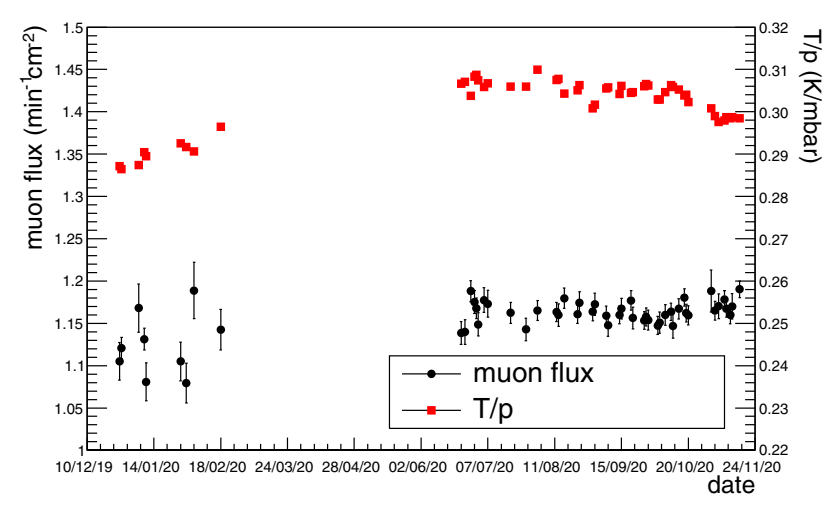

Figure 3. Cosmic ray muon flux and $T / p$ as a function of date.

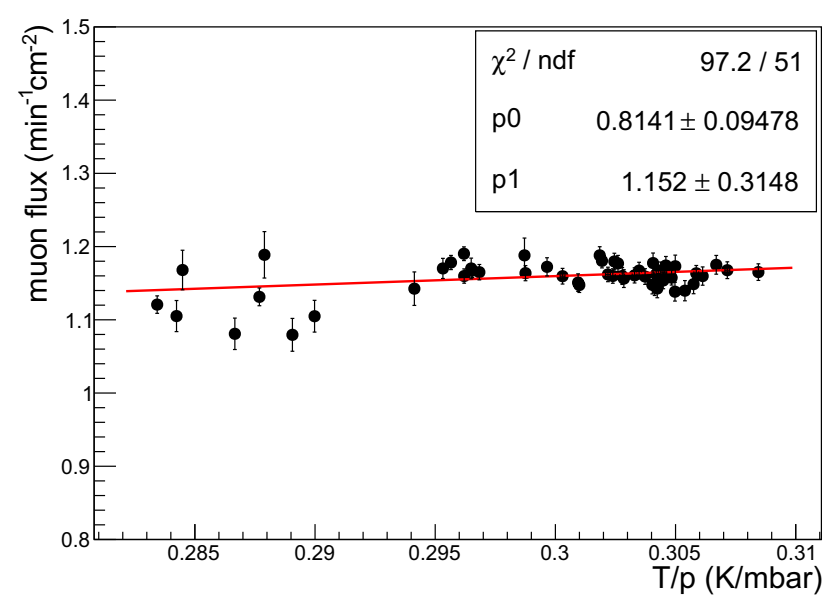

Figure 4. Correlation of cosmic muon flux with the ratio of temperature and pressure.

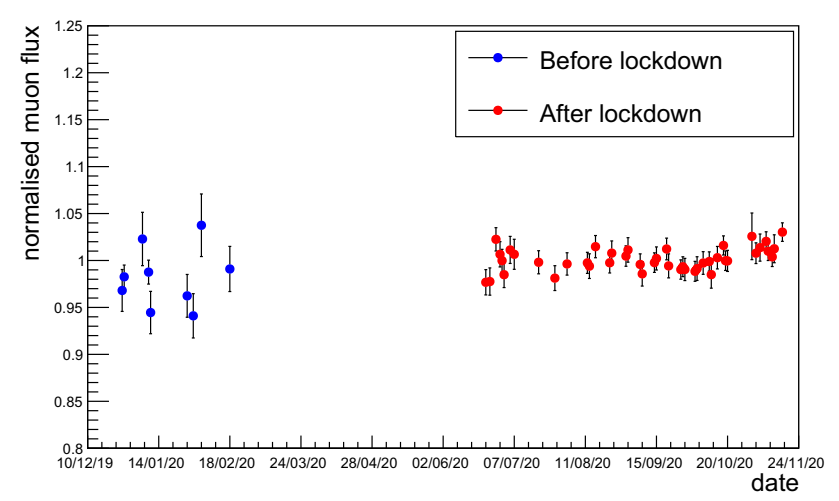

Figure 5. Normalised muon flux as a function of date. The gap between the two sets of data is the period of lockdown.

(threshold is $-15 \mathrm{mV}$ ). Each data point represents a 30-min long measurement. To check the health of the individual detectors, the single count rates of all the modules are measured several times. The single count rates of scintillators $\mathrm{SC} 1, \mathrm{SC} 2$ and $\mathrm{SC} 3$ are found to be $\sim 77, \sim 28$ and $\sim 171 \mathrm{~Hz}$ respectively.
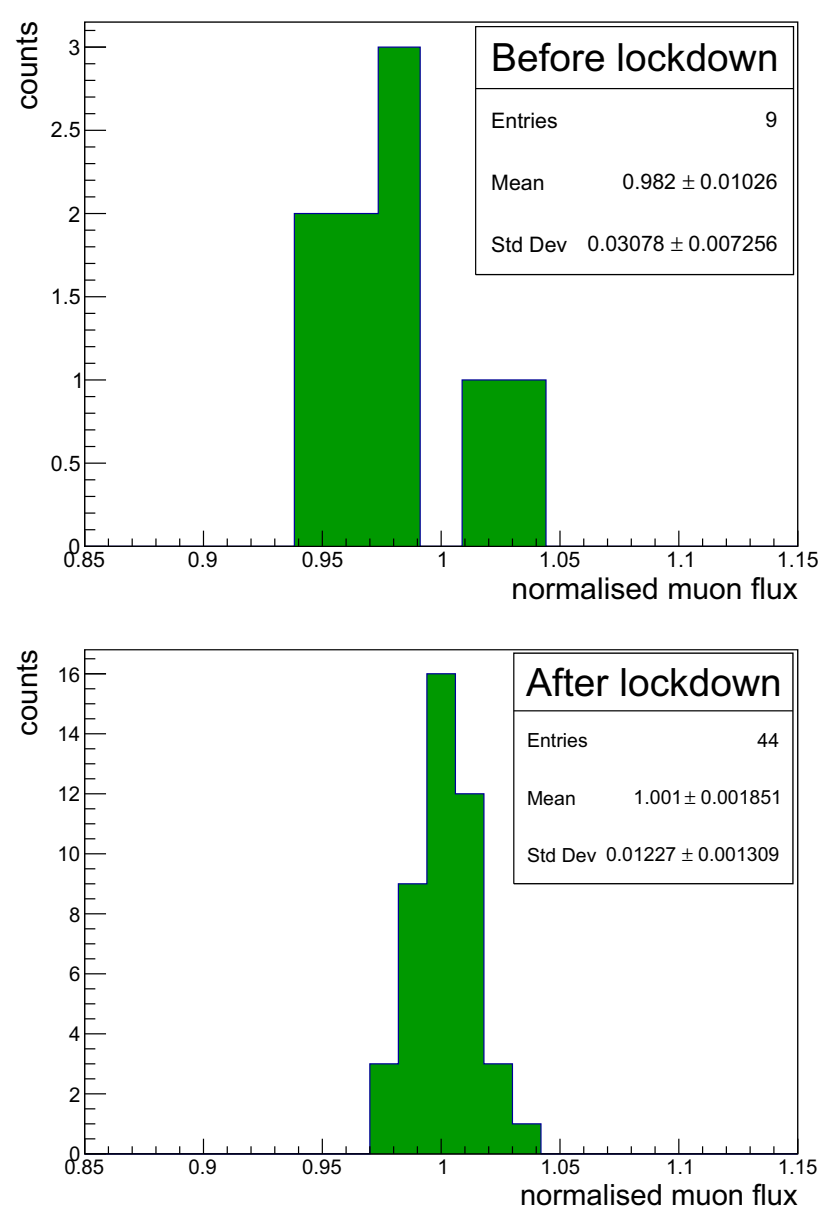

Figure 6. Distribution of normalised muon flux before and after the lockdown.

\section{Results}

The cosmic ray flux is measured in Kolkata before and after the lockdown due to the COVID-19 outbreak. The average muon flux before and after the lockdown is shown in figure 3 as a function of date. We had a very small amount of data before lockdown. The cosmic ray flux depends on atmospheric parameters like temperature and pressure $[9,10]$. In this work, the temperature and pressure data are collected from ref. [11]. The ratio of temperature and pressure as a function of date is also shown in figure 3 . In order to normalise the temperature $(T=t+273)$ and pressure $(p)$ effects, a simple correlation between cosmic muon flux and $T / p$ is studied using the relation $p 0+p 1(T / p)$. The correlation between muon flux and $T / p$ is shown in figure 4 . The parameters obtained from the correlation are $0.81 \pm 0.09$ $\mathrm{min}^{-1} \mathrm{~cm}^{-2}(p 0)$ and $1.15 \pm 0.31 \mathrm{~min}^{-1} \mathrm{~cm}^{-2} \mathrm{k}^{-1} \mathrm{mbar}$ ( $p 1)$ respectively. 

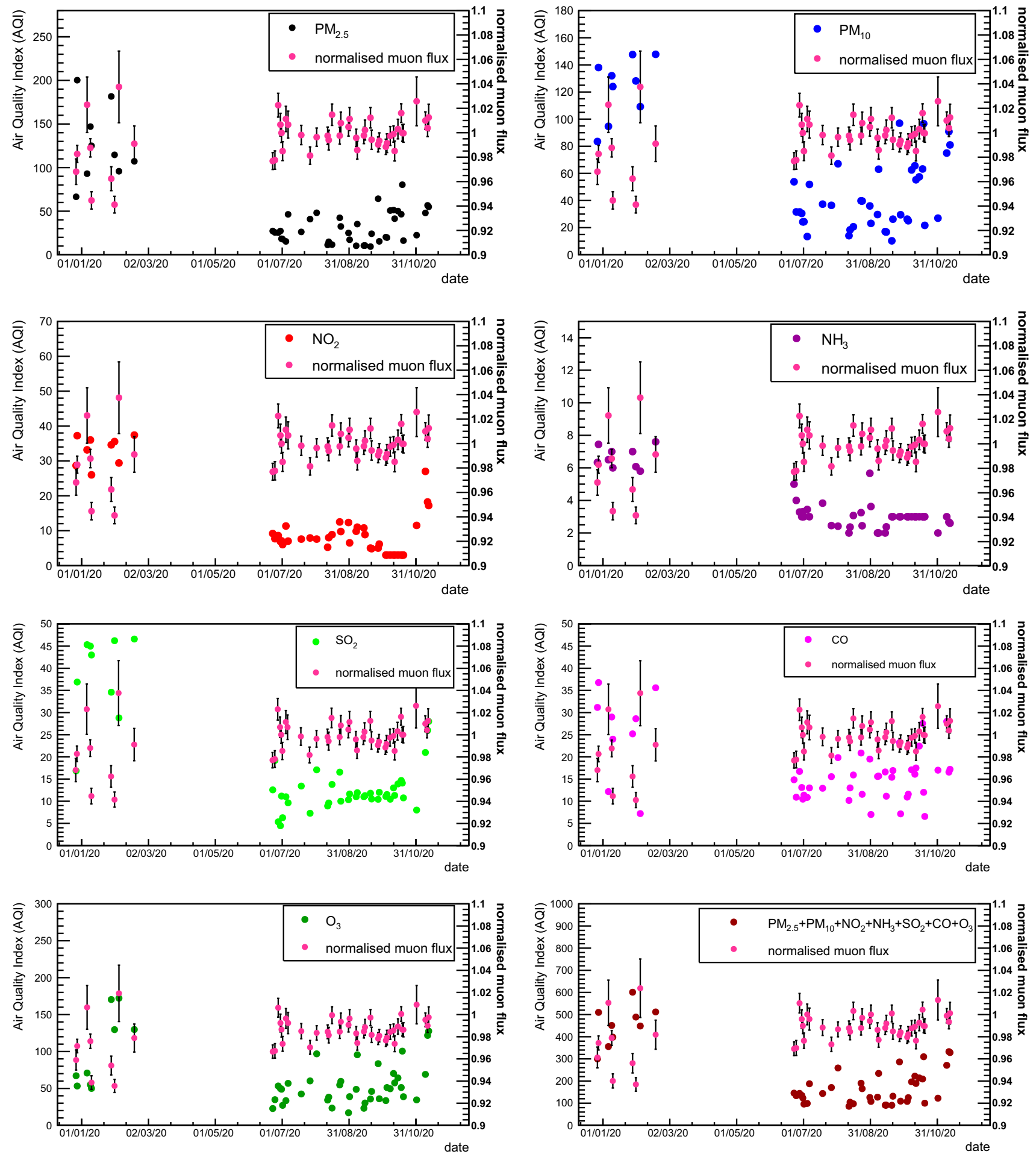

Figure 7. Average air quality index (AQI) of seven most abundant air pollutants measured at Bidhannagar, Kolkata station [6] and the normalised muon flux as a function of date.

A positive correlation is observed between the muon flux and $T / p$. Using the parameters, the muon flux measured before and after the lockdown is normalised and shown in figure 5.
In figure 6 , the distribution of the normalised muon flux is shown before and after the lockdown. It is found that the mean normalised muon flux before the lockdown period is 0.982 with a standard deviation of 0.031 , 

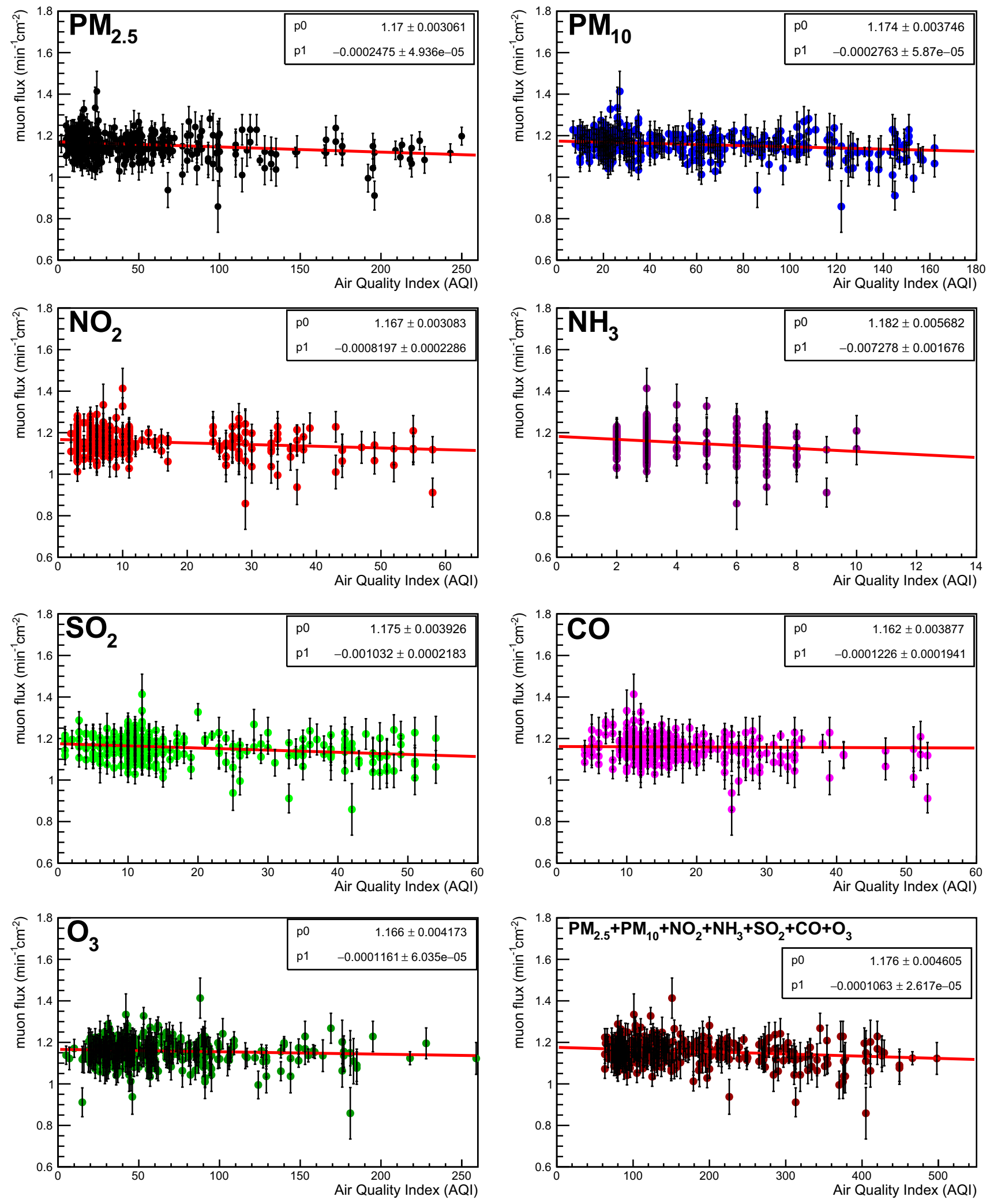

Figure 8. Measured muon flux as a function of AQI of seven most abundant air pollutants. 

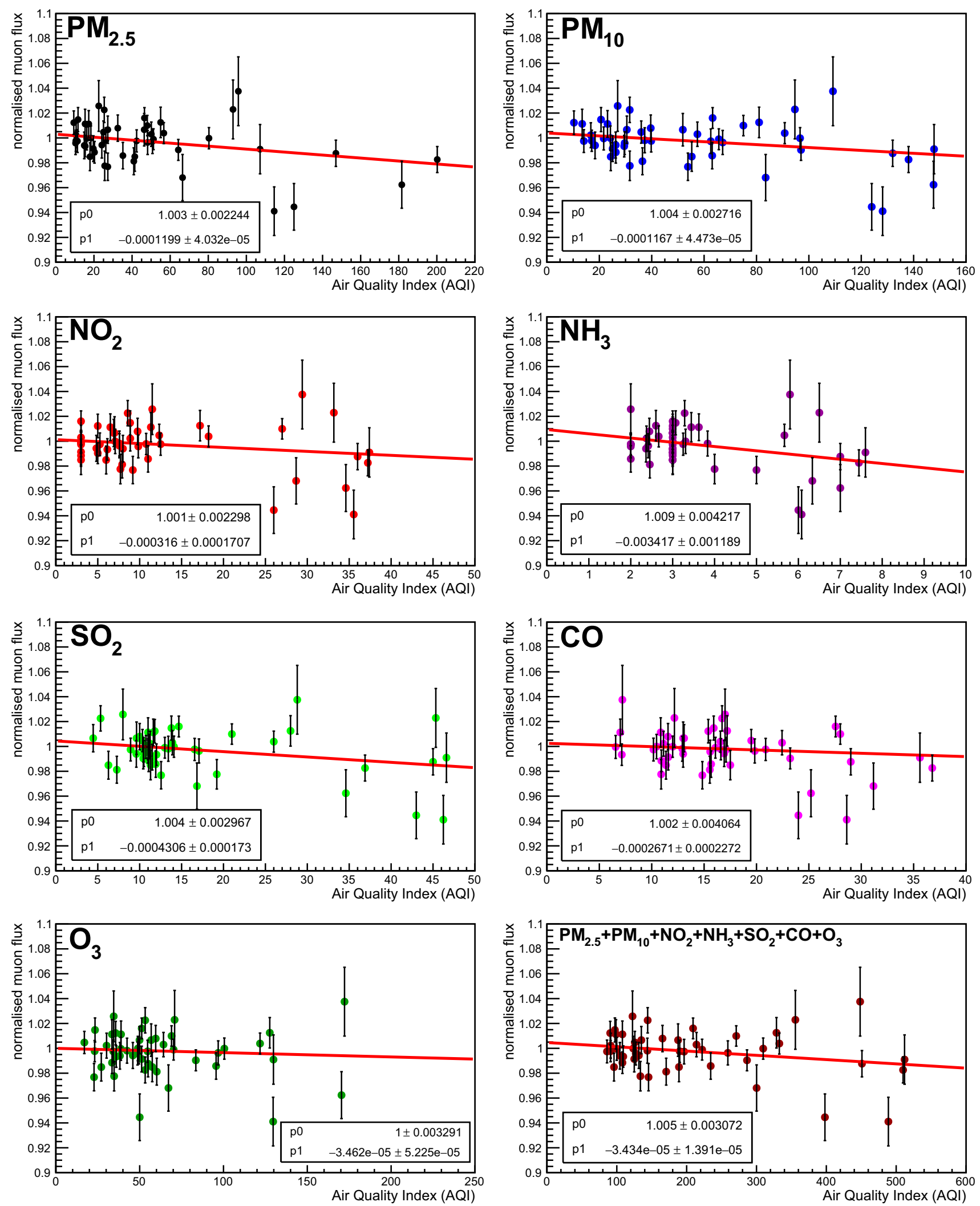

Figure 9. Normalised muon flux as a function of AQI of seven most abundant air pollutants. 
Table 1. Value of the fit parameters of the muon flux vs. AQI curve with seven most abundant air pollutants.

\begin{tabular}{lll}
\hline Air pollutant type & \multicolumn{1}{c}{$p 0$} & \multicolumn{1}{c}{$p 1$} \\
\hline $\mathrm{PM}_{2.5}$ & $1.17 \pm 0.003061$ & $-0.0002475 \pm 4.936 \mathrm{e}-05$ \\
$\mathrm{PM}_{10}$ & $1.174 \pm 0.003746$ & $-0.0002763 \pm 5.87 \mathrm{e}-05$ \\
$\mathrm{NO}_{2}$ & $1.167 \pm 0.003083$ & $-0.0008197 \pm 0.0002286$ \\
$\mathrm{NH}_{3}$ & $1.182 \pm 0.005682$ & $-0.007278 \pm 0.001676$ \\
$\mathrm{SO}_{2}$ & $1.175 \pm 0.003926$ & $-0.001032 \pm 0.0002183$ \\
$\mathrm{CO}$ & $1.162 \pm 0.003877$ & $-0.0001226 \pm 0.0001941$ \\
$\mathrm{O}_{3}$ & $1.166 \pm 0.004173$ & $-0.0001161 \pm 6.035 \mathrm{e}-05$ \\
Gross & $1.176 \pm 0.004605$ & $-0.0001063 \pm 2.617 \mathrm{e}-05$ \\
\hline
\end{tabular}

whereas that after the lockdown is 1.001 with a standard deviation of 0.012 , i.e. $1.9 \%$ increment in muon flux is found after the lockdown.

In figure 7, we have reported the individual air pollutants (average air quality index (AQI) which is proportional to the concentration of the pollutants) before and after the lockdown (on the dates of cosmic ray data recording) and a clear decrement in the concentrations of the air pollutants has been observed after the lockdown. One striking thing we observe during this study, is that the concentration of $\mathrm{O}_{3}$ also decreases after lockdown in our case unlike in ref. [2] where the concentration of $\mathrm{O}_{3}$ was reported to be increased during the lockdown (for a short time period though). Actually in ref. [2] the concentration of $\mathrm{O}_{3}$ was reported to be increased during the lockdown (March 25-April 6, 2020) compared to the values before the lockdown (10-20 March 2020). In ref. [6], the concentration values of $\mathrm{O}_{3}$ before the lockdown are quoted for December 27, 2019 to February 18, 2020 and after lockdown those are during June 24, 2020 and November 17, 2020. Clearly, the dates in refs $[2,6]$ are different and hence the discrepancy. Figure 8 represents the variation of the muon flux with AQI for the seven air pollutants individually and with the gross pollutants present. We observe a correlation between cosmic ray muon flux and the concentrations of the air pollutants before and after the lockdown where the flux increases with decrease in the concentrations of the air pollutants. The details of the variation found by linear fitting of the muon flux vs. air quality index curve for different pollutants, are tabulated in table 1.

$T / p$ normalised muon flux is also plotted as a function of the average AQI for the seven air pollutants individually and with the gross pollutants present in figure 9 . Here also we observed that the normalised flux increases with decrease in the concentrations of the air pollutants.

\section{Summary and discussion}

Cosmic ray muon flux was measured using the coincidence technique with plastic scintillation detectors. To restrict the outbreak of COVID-19, Government of India imposed 67 days of nationwide complete lockdown in three phases. After that, the unlocking was declared in phases in different parts of India. Before the lockdown, we collected some cosmic ray flux data. After lockdown the measurement was continued to compare with the flux measured before lockdown. In our measurement, it is found that the cosmic ray flux remained more or less unchanged before and after the lockdown. However, it is well known that atmospheric temperature and pressure affect the cosmic ray flux and we looked for any such possible correlation. A positive correlation is indeed observed between the muon flux and the ratio of atmospheric temperature and pressure. This correlation is fitted well by a function of the form $p 0+p 1(T / p)$, and the fit parameters $p 0$ and $p 1$ are used to normalise the $T / p$ effect on the cosmic muon flux. It is found that the mean normalised muon flux before and after the lockdown period are 0.982 with a standard deviation of 0.031 and 1.001 with a standard deviation of 0.012 respectively.

A lockdown such as the one implemented due to COVID-19 typically has significant influence on the atmospheric condition in terms of the presence of pollutants. We wanted to study any possible correlation of measured cosmic ray muon flux with this. To realise this, we considered the seven most abundant air pollutants $\left(\mathrm{PM}_{2.5}, \mathrm{PM}_{10}, \mathrm{NO}_{2}, \mathrm{NH}_{3}, \mathrm{SO}_{2}, \mathrm{CO}, \mathrm{O}_{3}\right)$ and investigated the change in their concentrations with date (before and after the lockdown). We found significant declination in the concentrations of the pollutants and we tried to look for any correlation with the measured muon flux within the stipulated time window. The result shows a clear correlation as with decreasing concentrations of the air pollutants we observed an increasing trend of the normalised muon flux. From our observation, one can comment that the increase in cosmic ray flux can also be considered as one of the secondary indicators of less polluted air.

However, there are a few limitations of our measurement. First, the detector coverage area was very small, 
resulting in low statistics. Second, the statistics of muon data before lockdown is small. It will be very interesting if any other research laboratory having a large facility of cosmic ray flux measurement can try to study such correlation.

\section{Acknowledgements}

The authors would like to thank Dr Abhijit Chatterjee, Prof. Sibaji Raha, Prof. Rajarshi Ray, Prof. Somshubhro Bandyopadhyay and Dr Sidharth K Prasad for valuable discussions and suggestions in the course of the study. We would also like to thank Mrs Sharmili Rudra, Dr Rama Prasad Adak, Mr Dipanjan Nag, Ms Nilanjana Nandi and Mr Subrata Das for helping in the fabrication of the detectors. This work is partially supported by the research grant SR/MF/PS-01/2014-BI from DST, Govt. of India, the research grant of CBM-MuCh project from BI-IFCC, DST, Govt. of India and IRHPA (Intensification of Research in High Priority Areas/Sanction No. IR/S2/PF.01/2011) scheme. A Sen acknowledges his Inspire Fellowship research grant (DST/INSPIRE Fellowship/2018/IF180361).

\section{References}

[1] V Valkovic̀, Radioactivity in the environment, https:// doi.org/10.1016/B978-044482954-2.50002-2 (2000) pp. 5-32

[2] S Jain et al, Aerosol Air Quality Res. 20, 1222 (2020)

[3] S Chen et al, https://doi.org/10.1371/journal.pone. 0215663 (2019)

[4] Abhijit Chatterjee et al, Atmospheric environment, https://doi.org/10.1016/j.atmosenv.2020.117947

[5] https://www.covid19india.org/

[6] https://app.cpcbccr.com/AQI_India/

[7] S Roy et al, Proceedings of ADNHEAP 2017, Springer Proceedings in Physics 2017, pp. 199-204, ISBN 978981-10-7664-0

[8] S Shaw et al, Proc. DAE Symp. Nucl. Phys. 62, 1030 (2017)

[9] M Neira, A Prüss-Ustün and P Mudu, Lancet 392, 1178 (2018)

[10] M Zazyan et al, J. Space Weather Space Clim. 5, A6 (2015)

[11] https://www.timeanddate.com/weather/india/kolkata 